New kinds of matrix polynomials

\author{
Bayram Çekim
}




\title{
NEW KINDS OF MATRIX POLYNOMIALS
}

\author{
BAYRAM ÇEKIM \\ Received 4 February, 2013
}

\begin{abstract}
In this study, we introduce new matrix poynomials and derive their properties such as explict representations, recurrence relations and generating matrix functions.
\end{abstract}

2010 Mathematics Subject Classification: 33C45; 15A60

Keywords: matrix polynomials, reccurrence relation, generating matrix functions

\section{INTRODUCTION}

Recently, mathematicians have interested in some properties of the orthogonal matrix polynomials. For example, Jódar and et. al have introduced and studied Laguerre matrix polynomials [8], Gegenbauer matrix polynomials [9], Hermite matrix polynomials [7], Chebyshev matrix polynomials [4] and Jacobi matrix polynomials [3]. Then the Konhauser matrix polynomials [11], the multivariable Humbert matrix polynomials [1] and the Bessel matrix polynomials [10] have been studied. Furthermore, one can find several papers concerning the orthogonal matrix polynomials (see [2,12]). In this paper, we define new matrix polynomials and derive some of their properties.

Throughout this paper, for a matrix $A$ in $\mathbb{C}^{r \times r}$, its spectrum $\sigma(A)$ denotes the set of all eigenvalues of $A$. Furthermore the identity matrix and the zero matrix of $\mathbb{C}^{r \times r}$ will be denoted by $I$ and $\mathbf{0}$, respectively. If $f(z)$ and $g(z)$ are holomorphic functions of the complex variable $z$, which are defined in an open set $\Omega$ of the complex plane and $A$ is a matrix in $\mathbb{C}^{r \times r}$ with $\sigma(A) \subset \Omega$, then from the properties of the matrix functional calculus in [6], it follows that:

$$
f(A) g(A)=g(A) f(A) .
$$

Let $A$ be a matrix in $\mathbb{C}^{r \times r}$ satisfying $(-k) \notin \sigma(A)$ for $k \in \mathbb{Z}^{+}$and $\lambda$ be a complex number whose real part is positive. Then the Laguerre matrix polynomials $L_{n}^{(A, \lambda)}(x)$ are defined by [8]:

$$
L_{n}^{(A, \lambda)}(x)=\sum_{k=0}^{n} \frac{(-1)^{k}}{k !(n-k) !}(A+I)_{n}\left[(A+I)_{k}\right]^{-1}(\lambda x)^{k}, n \in \mathbb{N} .
$$


Also Laguerre matrix polynomials have the following generating matrix function:

$$
\sum_{n=0}^{\infty} L_{n}^{(A, \lambda)}(x) t^{n}=(1-t)^{-A-I} e^{-\frac{\lambda x t}{1-t}} ; x \in \mathbb{C}, t \in \mathbb{C},|t|<1 .
$$

\section{DEFINITIONS AND PROPERTIES OF NEW MATRIX POLYNOMIALS}

In this section, we define two new matrix polynomials $\left\{f_{n, m}^{(A, \lambda)}(x)\right\}_{n=0}^{\infty}$ and $\left\{g_{n, m}^{(A, \lambda)}(x)\right\}_{n=0}^{\infty}$ via following generating matrix functions

$$
\begin{aligned}
& F^{(A, \lambda)}(x, t)=\left(1-t^{m}\right)^{-A} e^{-\frac{x t \lambda}{1-t^{m}}}=\sum_{n=0}^{\infty} f_{n, m}^{(A, \lambda)}(x) t^{n} ;|t|<1 \\
& G^{(A, \lambda)}(x, t)=\left(1+t^{m}\right)^{-A} e^{-\frac{x t \lambda}{1+t^{m}}}=\sum_{n=0}^{\infty} g_{n, m}^{(A, \lambda)}(x) t^{n} ;|t|<1
\end{aligned}
$$

where $A$ is a matrix in $\mathbb{C}^{r \times r}, m$ is a positive natural number and $\lambda$ is an arbitrary number, respectively. Here, (2.1) and (2.2) are matrix versions of polynomials given in [5].

Using (2.1) and (2.2), we obtain the following explicit representations for new matrix polynomials

$$
\begin{aligned}
& f_{n, m}^{(A, \lambda)}(x)=\sum_{i=0}^{\left[\frac{n}{m}\right]} \frac{(-1)^{n-m i}(A+(n-m i) I)_{i}}{(n-m i) ! i !}(\lambda x)^{n-m i}, \\
& g_{n, m}^{(A, \lambda)}(x)=\sum_{i=0}^{\left[\frac{n}{m}\right]} \frac{(-1)^{n-(m-1) i}(A+(n-m i) I)_{i}}{(n-m i) ! i !}(\lambda x)^{n-m i}
\end{aligned}
$$

where [.] denotes the integer part. Taking $m=1$ in (2.3), (2.3) reduces Laguerre matrix polynomials as follows:

$$
\begin{aligned}
f_{n, 1}^{(A, \lambda)}(x) & =\sum_{i=0}^{n} \frac{(-1)^{n-i}(A+(n-i) I)_{i}}{(n-i) ! i !}(\lambda x)^{n-i} \\
& =\sum_{i=0}^{n} \frac{(-1)^{i}(A)_{n}(A)_{i}^{-1}}{(n-i) ! i !}(\lambda x)^{i}=L_{n}^{(A-I, \lambda)}(x)
\end{aligned}
$$

where

$\alpha \notin \mathbb{Z}^{-} \cup\{0\}$ for $\forall \alpha \in \sigma(A)$ and $\lambda$ be a complex parameter with $\operatorname{Re}(\lambda)>0$ (2.4) (see [8]). 
Differentiating (2.1) with respect to $x$, the $f_{n, m}^{(A, \lambda)}(x)$ matrix polynomials satisfy recurrence relations

$$
\frac{\partial f_{n, m}^{(A, \lambda)}(x)}{\partial x}=-\lambda f_{n-1, m}^{(A+I, \lambda)}(x)
$$

and a generalization of the above equation

$$
\frac{\partial^{k} f_{n, m}^{(A, \lambda)}(x)}{\partial x^{k}}=(-\lambda)^{k} f_{n-k, m}^{(A+k I, \lambda)}(x) ; n \geq k .
$$

For the special case $m=1$ in (2.5), it follows that

$$
\frac{\partial L_{n}^{(A-I, \lambda)}(x)}{\partial x}=-\lambda L_{n-1}^{(A, \lambda)}(x)
$$

where $A$ and $\lambda$ satisfy (2.4). Similarly, for $g_{n, m}^{(A, \lambda)}(x)$, we have

$$
\begin{aligned}
\frac{\partial g_{n, m}^{(A, \lambda)}(x)}{\partial x} & =-\lambda g_{n-1, m}^{(A+I, \lambda)}(x) \\
\frac{\partial^{k} g_{n, m}^{(A, \lambda)}(x)}{\partial x^{k}} & =(-\lambda)^{k} g_{n-k, m}^{(A+k I, \lambda)}(x) ; n \geq k .
\end{aligned}
$$

On the other hand, differentiating (2.1) with respect to $t$, the $f_{n, m}^{(A, \lambda)}(x)$ matrix polynomials satisfy recurrence relation

$$
\begin{aligned}
n f_{n, m}^{(A, \lambda)}(x)- & (n-m) f_{n-m, m}^{(A, \lambda)}(x)=m A\left[f_{n-m, m}^{(A+I, \lambda)}(x)-f_{n-2 m, m}^{(A+I, \lambda)}(x)\right] \\
- & x \lambda\left[f_{n-1, m}^{(A+I, \lambda)}(x)+(m-1) f_{n-m-1, m}^{(A+I, \lambda)}(x)\right]
\end{aligned}
$$

where $n \geq 2 m$. For the special case $m=1$ in (2.6), it holds that

$$
n L_{n}^{(A-I, \lambda)}(x)-(n-1) L_{n-1}^{(A-I, \lambda)}(x)=A\left[L_{n-1}^{(A, \lambda)}(x)-L_{n-2}^{(A, \lambda)}(x)\right]-x \lambda L_{n-1}^{(A, \lambda)}(x)
$$

where $n \geq 2, A$ and $\lambda$ satisfy (2.4). For $f_{n, m}^{(A, \lambda)}(x)$, other recurrence relation via differentiating (2.1) with respect to $t$ is

$$
\begin{aligned}
n f_{n, m}^{(A, \lambda)}(x) & =[m(A-2 I)+2 n I] f_{n-m, m}^{(A, \lambda)}(x)-[m(A-2 I)+n I] f_{n-2 m, m}^{(A, \lambda)}(x) \\
& -x \lambda\left[f_{n-1, m}^{(A, \lambda)}(x)+(m-1) f_{n-m-1, m}^{(A, \lambda)}(x)\right]
\end{aligned}
$$

where $n \geq 2 m$. For $m=1$, (2.7) reduces the recurrence relation satisfied by the Laguerre matrix polynomials as follows:

$n L_{n}^{(A, \lambda)}(x)=[(A-I)+2 n I-x \lambda I] L_{n-1}^{(A, \lambda)}(x)-[(A-I)+n I] L_{n-2}^{(A, \lambda)}(x), n \geq 2$

where $A$ and $\lambda$ satisfy (2.4). On the one hand, for $g_{n, m}^{(A, \lambda)}(x)$, we derive the following relations:

$$
n g_{n, m}^{(A, \lambda)}(x)=-m A g_{n-m, m}^{(A+I, \lambda)}(x)-x \lambda g_{n-1, m}^{(A+2 I, \lambda)}(x)+(m-1) x \lambda g_{n-m-1, m}^{(A+2 I, \lambda)}(x)
$$


where $n \geq m+1$ and

$$
\begin{aligned}
& {[2(n-m) I+m A] g_{n-m, m}^{(A, \lambda)}(x)+[(n-2 m) I+m A] g_{n-2 m, m}^{(A, \lambda)}(x)} \\
& =-x \lambda g_{n-1, m}^{(A, \lambda)}(x)+x \lambda(m-1) g_{n-m-1, m}^{(A, \lambda)}(x)-n g_{n, m}^{(A, \lambda)}(x)
\end{aligned}
$$

where $n \geq 2 m$. Now, we shall prove interesting relations for $f_{n, m}^{(A, \lambda)}(x)$ and $g_{n, m}^{(A, \lambda)}(x)$. Starting from (2.1), we have

$$
F^{(A, \lambda)}(x, t) F^{(A, \lambda)}(y, t)=\left(1-t^{m}\right)^{-2 A} e^{-\frac{(x+y) t \lambda}{1-t^{m}}}=\sum_{n=0}^{\infty} f_{n, m}^{(2 A, \lambda)}(x+y) t^{n} .
$$

Using (2.1) in the left-hand side of (2.8), it follows that

$$
\sum_{i=0}^{n} f_{n-i, m}^{(A, \lambda)}(x) f_{i, m}^{(A, \lambda)}(y)=f_{n, m}^{(2 A, \lambda)}(x+y)
$$

For $m=1,(2.9)$ reduces

$$
\sum_{i=0}^{n} L_{n-i}^{(A-I, \lambda)}(x) L_{i}^{(A-I, \lambda)}(y)=L_{n}^{(2 A-I, \lambda)}(x+y)
$$

where $A$ and $\lambda$ satisfy (2.4) and $\mu \notin \mathbb{Z}^{-} \cup\{0\}$ for $\forall \mu \in \sigma(2 A)$. In the same way, $g_{n, m}^{(A, \lambda)}(x)$ matrix polynomials satisfy

$$
\sum_{i=0}^{n} g_{n-i, m}^{(A, \lambda)}(x) g_{i, m}^{(A, \lambda)}(y)=g_{n, m}^{(2 A, \lambda)}(x+y) .
$$

Similarly, we have

$$
\begin{aligned}
& \sum_{i=0}^{n} g_{n-i, m}^{(A, \lambda)}(x) g_{i, m}^{(B, \lambda)}(x)=g_{n, m}^{(A+B, \lambda)}(2 x), \\
& \sum_{i=0}^{n} f_{n-i, m}^{(A, \lambda)}(x) f_{i, m}^{(B, \lambda)}(x)=f_{n, m}^{(A+B, \lambda)}(2 x)
\end{aligned}
$$

where $A B=B A$. For $m=1$ in (2.10), we get

$$
\sum_{i=0}^{n} L_{n-i}^{(A-I, \lambda)}(x) L_{i}^{(B-I, \lambda)}(x)=L_{n}^{(A+B-I, \lambda)}(2 x)
$$

where $A$ and $\lambda$ satisfy (2.4), $v \notin \mathbb{Z}^{-} \cup\{0\}$ for $\forall v \in \sigma(B)$ and $\eta \notin \mathbb{Z}^{-} \cup\{0\}$ for $\forall \eta \in \sigma(A+B)$. On the other hand, (2.8) can be written as

$$
\left(1-t^{m}\right)^{-2 A} e^{-\frac{x t \lambda}{1-t^{m}}}=e^{\frac{y t \lambda}{1-t^{m}}} \sum_{i=0}^{\infty} f_{i, m}^{(2 A, \lambda)}(x+y) t^{i}
$$


and then

$$
\begin{aligned}
\sum_{n=0}^{\infty} f_{n, m}^{(2 A, \lambda)}(x) t^{n} & =\left[\sum_{n=0}^{\infty} \frac{\left(\frac{y t \lambda}{1-t^{m}}\right)^{n}}{n !}\right]\left[\sum_{i=0}^{\infty} f_{i, m}^{(2 A, \lambda)}(x+y) t^{i}\right] \\
& =\left[\sum_{n=0}^{\infty} \frac{(y t \lambda)^{n}}{n !}\right]\left[\sum_{j=0}^{\infty} \frac{(n) j}{j !} t^{m j}\right]\left[\sum_{i=0}^{\infty} f_{i, m}^{(2 A, \lambda)}(x+y) t^{i}\right] \\
& =\sum_{n=0}^{\infty} \sum_{j=0}^{\left[\frac{n}{m}\right]} \sum_{i=0}^{n-m j} \frac{\lambda^{n-m j-i} y^{n-m j-i}}{(n-m j-i) !} \frac{(n-m j-i)_{j}}{j !} f_{i, m}^{(2 A, \lambda)}(x+y) t^{n}
\end{aligned}
$$

Comparing the coefficients of $t^{n}$, we have

$$
f_{n, m}^{(2 A, \lambda)}(x)=\sum_{j=0}^{\left[\frac{n}{m}\right]} \sum_{i=0}^{n-m j} \frac{\lambda^{n-m j-i} y^{n-m j-i}}{(n-m j-i) !} \frac{(n-m j-i)_{j}}{j !} f_{i, m}^{(2 A, \lambda)}(x+y) .
$$

If $m=1$ in (2.11), we obtain

$$
L_{n}^{(2 A-I, \lambda)}(x)=\sum_{j=0}^{n} \sum_{i=0}^{n-j} \frac{\lambda^{n-j-i} y^{n-j-i}}{(n-j-i) !} \frac{(n-j-i)_{j}}{j !} L_{i}^{(2 A-I, \lambda)}(x+y)
$$

where $\mu \notin \mathbb{Z}^{-} \cup\{0\}$ for $\forall \mu \in \sigma(2 A)$. Similarly, for $g_{n, m}^{(A, \lambda)}(x)$, we get

$$
g_{n, m}^{(2 A, \lambda)}(x)=\sum_{j=0}^{\left[\frac{n}{m}\right]} \sum_{i=0}^{n-m j} \frac{(-1)^{j} \lambda^{n-m j-i} y^{n-m j-i}}{(n-m j-i) !} \frac{(n-m j-i)_{j}}{j !} g_{i, m}^{(2 A, \lambda)}(x+y) .
$$

Now, let's give relations for the derivatives of new matrix polynomials. From (2.1), it follows that

$$
\frac{\partial^{s}}{\partial x^{s}} F^{(A, \lambda)}(x, t)=(-1)^{s} t^{s} \lambda^{s}\left(1-t^{m}\right)^{-A-s I} e^{-\frac{x t \lambda}{1-t^{m}}} .
$$

By the above equation and (2.1), we can write

$$
\begin{aligned}
\frac{\partial^{s}}{\partial x^{s}} F^{(A, \lambda)}(x, t) \frac{\partial^{s}}{\partial y^{s}} F^{(A, \lambda)}(y, t) & =\sum_{n=0}^{\infty} \lambda^{2 s} f_{n, m}^{(2 A+2 s I, \lambda)}(x+y) t^{n+2 s}, \\
\frac{\partial^{s}}{\partial x^{s}}\left[\sum_{n=0}^{\infty} f_{n, m}^{(A, \lambda)}(x) t^{n}\right] \frac{\partial^{s}}{\partial y^{s}}\left[\sum_{i=0}^{\infty} f_{i, m}^{(A, \lambda)}(y) t^{i}\right] & =\sum_{n=0}^{\infty} \lambda^{2 s} f_{n, m}^{(2 A+2 s I, \lambda)}(x+y) t^{n+2 s}, \\
\sum_{n=0}^{\infty} \sum_{i=0}^{n} D_{x}^{s} f_{n-i, m}^{(A, \lambda)}(x) D_{y}^{s} f_{i, m}^{(A, \lambda)}(y) t^{n} & =\sum_{n=2 s}^{\infty} \lambda^{2 s} f_{n-2 s, m}^{(2 A+2 s I, \lambda)}(x+y) t^{n}
\end{aligned}
$$


where $D_{x}^{s}=\frac{d^{s}}{d x^{s}}$ and $D_{y}^{s}=\frac{d^{s}}{d y^{s}}$. Comparing the coefficients of $t^{n}$, we have

$$
\sum_{i=0}^{n} D_{x}^{s} f_{n-i, m}^{(A, \lambda)}(x) D_{y}^{s} f_{i, m}^{(A, \lambda)}(y)=\lambda^{2 s} f_{n-2 s, m}^{(2 A+2 s I, \lambda)}(x+y) ; n \geq 2 s .
$$

If $m=1$ in (2.12), (2.12) reduces

$$
\sum_{i=0}^{n} D_{x}^{s} L_{n-i}^{(A-I, \lambda)}(x) D_{y}^{s} L_{i}^{(A-I, \lambda)}(y)=\lambda^{2 s} L_{n-2 s}^{(2 A+(2 s-1) I, \lambda)}(x+y) \quad ; n \geq 2 s
$$

where $A$ and $\lambda$ satisfy (2.4) and $\mu \notin \mathbb{Z}^{-} \cup\{0\}$ for $\forall \mu \in \sigma(2 A)$. In the same way, we get

$$
\sum_{i=0}^{n} D_{x}^{s} g_{n-i, m}^{(A, \lambda)}(x) D_{y}^{s} g_{i, m}^{(A, \lambda)}(y)=\lambda^{2 s} g_{n-2 s, m}^{(2 A+2 s I, \lambda)}(x+y) \quad ; n \geq 2 s .
$$

On the one hand, using (2.1) and (2.2), we can write

$$
\frac{\partial^{k}}{\partial x^{k}} F^{(A, \lambda)}(x, t) \frac{\partial^{k}}{\partial x^{k}} G^{(A, \lambda)}(x, t)=\sum_{n=0}^{\infty} \lambda^{2 k} f_{n, 2 m}^{(A+k I, \lambda)}(2 x) t^{n+2 k} .
$$

Thus, it holds that

$$
\sum_{i=0}^{n} D_{x}^{k} f_{n-i, m}^{(A, \lambda)}(x) D_{x}^{k} g_{i, m}^{(A, \lambda)}(x)=\lambda^{2 k} f_{n-2 k, 2 m}^{(A+k I, \lambda)}(2 x) ; n \geq 2 k .
$$

The generalizations of (2.10) are as follows:

$$
\begin{aligned}
\sum_{i_{1}+i_{2}+\ldots+i_{k}=n} f_{i_{1}, m}^{\left(A_{1}, \lambda\right)}\left(x_{1}\right) \ldots f_{i_{k}, m}^{\left(A_{k}, \lambda\right)}\left(x_{k}\right) & =f_{n, m}^{\left(A_{1}+\ldots+A_{k}, \lambda\right)}\left(x_{1}+\ldots+x_{k}\right), \\
\sum_{i_{1}+i_{2}+\ldots+i_{k}=n} g_{i_{1}, m}^{\left(A_{1}, \lambda\right)}\left(x_{1}\right) \ldots g_{i_{k}, m}^{\left(A_{k}, \lambda\right)}\left(x_{k}\right) & =g_{n, m}^{\left(A_{1}+\ldots+A_{k}, \lambda\right)}\left(x_{1}+\ldots+x_{k}\right) .
\end{aligned}
$$

where the matrices $A_{1}, \ldots, A_{k}$ are assumed to be commutative. For $k=0$ in (2.14), we have

$$
\sum_{i=0}^{n} f_{n-i, m}^{(A, \lambda)}(x) g_{i, m}^{(A, \lambda)}(x)=f_{n, m}^{(A, \lambda)}(2 x) .
$$

The generalization of (2.15) is

$$
\begin{aligned}
& \sum_{s=0}^{n}\left\{\sum_{i_{1}+i_{2}+\ldots+i_{k}=n-s} f_{i_{1}, m}^{\left(A_{1}, \lambda\right)}\left(x_{1}\right) \ldots f_{i_{k}, m}^{\left(A_{k}, \lambda\right)}\left(x_{k}\right)\right. \\
& \left.\times \sum_{j_{1}+j_{2}+\ldots+j_{k}=n} g_{j_{1}, m}^{\left(A_{1}, \lambda\right)}\left(x_{1}\right) \ldots g_{j_{k}, m}^{\left(A_{k}, \lambda\right)}\left(x_{k}\right)\right\}
\end{aligned}
$$




$$
=\sum_{i_{1}+i_{2}+\ldots+i_{k}=n} f_{i_{1}, 2 m}^{\left(A_{1}, \lambda\right)}\left(2 x_{1}\right) \ldots f_{i_{k}, 2 m}^{\left(A_{k}, \lambda\right)}\left(2 x_{k}\right)
$$

where the matrices $A_{1}, \ldots, A_{k}$ are assumed to be commutative.

\section{MultilineAR AND MULTILATERAL GENERATING MATRIX FUNCTIONS FOR}

$$
f_{n, m}^{(A, \lambda)}(x) \operatorname{AND} g_{n, m}^{(A, \lambda)}(x)
$$

In this section, we derive several families of bilinear and bilateral generating matrix functions for the new matrix polynomials generated by (2.1) and (2.2). We first state our result.

Theorem 1. Corresponding to a non-vanishing function $\Omega_{\mu}\left(y_{1}, \ldots, y_{s}\right)$ of $s$ complex variables $y_{1}, \ldots, y_{s}(s \in \mathbb{N})$ and of complex order $\mu$, let

$$
\Lambda_{\mu, v}\left(y_{1}, \ldots, y_{s} ; z\right):=\sum_{k=0}^{\infty} a_{k} \Omega_{\mu+v k}\left(y_{1}, \ldots, y_{s}\right) z^{k} ;\left(a_{k} \neq 0, \mu, v \in \mathbb{C}\right)
$$

and

$$
\Theta_{n, p, \mu, \nu}\left(x ; y_{1}, \ldots, y_{s} ; \zeta\right):=\sum_{k=0}^{[n / p]} a_{k} f_{n-p k, m}^{(A, \lambda)}(x) \Omega_{\mu+\nu k}\left(y_{1}, \ldots, y_{s}\right) \zeta^{k}
$$

where $n, p \in \mathbb{N}$ and (as usual) $[\alpha]$ represents the greatest integer in $\alpha \in \mathbb{R}$. Then we have

$$
\sum_{n=0}^{\infty} \Theta_{n, p, \mu, \nu}\left(x ; y_{1}, \ldots, y_{s} ; \frac{\eta}{t^{p}}\right) t^{n}=\left(1-t^{m}\right)^{-A} e^{-\frac{x t \lambda}{1-t^{m}}} \Lambda_{\mu, \nu}\left(y_{1}, \ldots, y_{s} ; \eta\right)
$$

provided that each member of (3.3) exists for $|t|<1$ and $\operatorname{Re}(\lambda)>0$.

Proof. For convenience, let $S$ denote the first member of the assertion (3.3) of Theorem 1 . Then, upon substituting for the polynomials

$\Theta_{n, p, \mu, \nu}\left(x ; y_{1}, \ldots, y_{s} ; \frac{\eta}{t^{p}}\right)$ from the definition (3.2) into the left-hand side of (3.3), we obtain

$$
S=\sum_{n=0}^{\infty} \sum_{k=0}^{[n / p]} a_{k} f_{n-p k, m}^{(A, \lambda)}(x) \Omega_{\mu+v k}\left(y_{1}, \ldots, y_{s}\right) \eta^{k} t^{n-p k} .
$$

Upon inverting the order of summation in (3.4), if we replace $n$ by $n+p k$, we can write

$$
\begin{aligned}
S & =\sum_{n=0}^{\infty} \sum_{k=0}^{\infty} a_{k} f_{n, m}^{(A, \lambda)}(x) \Omega_{\mu+v k}\left(y_{1}, \ldots, y_{s}\right) \eta^{k} t^{n} \\
& =\left(\sum_{n=0}^{\infty} f_{n, m}^{(A, \lambda)}(x) t^{n}\right)\left(\sum_{k=0}^{\infty} a_{k} \Omega_{\mu+v k}\left(y_{1}, \ldots, y_{s}\right) \eta^{k}\right)
\end{aligned}
$$




$$
=\left\{\left(1-t^{m}\right)^{-A} e^{-\frac{x t \lambda}{1-t^{m}}}\right\} \Lambda_{\mu, \nu}\left(y_{1}, \ldots, y_{s} ; \eta\right),
$$

which completes the proof of Theorem 1.

Corollary 1. Corresponding to a non-vanishing function $\Omega_{\mu}\left(y_{1}, \ldots, y_{s}\right)$ of s complex variables $y_{1}, \ldots, y_{s}(s \in \mathbb{N})$ and of complex order $\mu$, let

$$
\Lambda_{\mu, v}\left(y_{1}, \ldots, y_{s} ; z\right):=\sum_{k=0}^{\infty} a_{k} \Omega_{\mu+v k}\left(y_{1}, \ldots, y_{s}\right) z^{k} ;\left(a_{k} \neq 0, \mu, v \in \mathbb{C}\right)
$$

and

$$
\Theta_{n, p, \mu, \nu}\left(x ; y_{1}, \ldots, y_{s} ; \zeta\right):=\sum_{k=0}^{[n / p]} a_{k} g_{n-p k, m}^{(A, \lambda)}(x) \Omega_{\mu+\nu k}\left(y_{1}, \ldots, y_{s}\right) \zeta^{k}
$$

where $n, p \in \mathbb{N}$ and $[\alpha]$ represents the greatest integer in $\alpha \in \mathbb{R}$. Then we have

$$
\sum_{n=0}^{\infty} \Theta_{n, p, \mu, v}\left(x ; y_{1}, \ldots, y_{s} ; \frac{\eta}{t^{p}}\right) t^{n}=\left(1+t^{m}\right)^{-A} e^{-\frac{x t \lambda}{1+t^{m}}} \Lambda_{\mu, v}\left(y_{1}, \ldots, y_{s} ; \eta\right)
$$

provided that each member of (3.7) exists for $|t|<1$ and $\operatorname{Re}(\lambda)>0$.

By expressing the multivariable function $\Omega_{\mu+\nu k}\left(y_{1}, \ldots, y_{s}\right)\left(k \in \mathbb{N}_{0}, s \in \mathbb{N}\right)$ in terms of simpler function of one and more variables, we can give further applications of Theorem 1. For example, if we set $s=1$ and $\Omega_{\mu+v k}(y)=g_{\mu+v k, m}^{(B, \gamma)}(y)$ in Theorem 1, where $g_{k, m}^{(B, \gamma)}(y)$ is defined by (2.2), then we obtain the following result which provides a class of bilateral generating functions for the $f_{n, m}^{(A, \lambda)}(x)$ and $g_{n, m}^{(A, \lambda)}(x)$.

Corollary 2. If $\Lambda_{\mu, \nu}(y ; z):=\sum_{k=0}^{\infty} a_{k} g_{\mu+\nu k, m}^{(B, \gamma)}(y) z^{k}$ where $\left(a_{k} \neq 0, \mu, v \in \mathbb{N}_{0}\right)$; and

$$
\Theta_{n, p, \mu, v}(x ; y ; \zeta):=\sum_{k=0}^{[n / p]} a_{k} f_{n-p k, m}^{(A, \lambda)}(x) g_{\mu+v k, m}^{(B, \gamma)}(y) \zeta^{k}
$$

where $n, p \in \mathbb{N}$, then we have

$$
\sum_{n=0}^{\infty} \Theta_{n, p, \mu, \nu}\left(x ; y ; \frac{\eta}{t^{p}}\right) t^{n}=\left(1-t^{m}\right)^{-A} e^{-\frac{x t \lambda}{1-t^{m}}} \Lambda_{\mu, \nu}(y ; \eta)
$$

provided that each member of (3.8) exists. 
Remark 1. Using the generating relation (2.2) for $g_{k, m}^{(B, \gamma)}(y)$ and taking $a_{k}=1$, $\mu=0, v=1$, we have

$$
\begin{aligned}
& \sum_{n=0}^{\infty} \sum_{k=0}^{[n / p]} f_{n-p k, m}^{(A, \lambda)}(x) g_{k, m}^{(B, \gamma)}(y) \eta^{k} t^{n-p k} \\
& =\left(1-t^{m}\right)^{-A} e^{-\frac{x t \lambda}{1-t^{m}}}\left(1+\eta^{m}\right)^{-B} e^{-\frac{y \eta \gamma}{1+\eta^{m}}}
\end{aligned}
$$

where $|\eta|<1$.

Choosing $s=1$ and $\Omega_{\mu+v k}(y)=f_{\mu+\nu k, m}^{(B, \gamma)}(y),\left(\mu, v \in \mathbb{N}_{0}\right)$, in Theorem 1, we obtain the following class of bilinear generating function for the $f_{n, m}^{(A, \lambda)}(x)$.

Corollary 3. If $\Lambda_{\mu, v}(y ; z):=\sum_{k=0}^{\infty} a_{k} f_{\mu+\nu k, m}^{(B, \gamma)}(y) z^{k}$ where $\left(a_{k} \neq 0, \mu, v \in \mathbb{N}_{0}\right)$; and

$$
\Theta_{n, p, \mu, v}(x ; y ; \zeta):=\sum_{k=0}^{[n / p]} a_{k} f_{n-p k, m}^{(A, \lambda)}(x) f_{\mu+\nu k, m}^{(B, \gamma)}(y) \zeta^{k}
$$

where $n, p \in \mathbb{N}$, then we have

$$
\sum_{n=0}^{\infty} \Theta_{n, p, \mu, \nu}\left(x ; y ; \frac{\eta}{t^{p}}\right) t^{n}=\left(1-t^{m}\right)^{-A} e^{-\frac{x t \lambda}{1-t^{m}}} \Lambda_{\mu, \nu}(y ; \eta)
$$

provided that each member of (3.9) exists.

Remark 2. Using Corollary 3 and taking $a_{k}=1, \mu=0, v=1$, we have

$$
\begin{aligned}
& \sum_{n=0}^{\infty} \sum_{k=0}^{[n / p]} f_{n-p k, m}^{(A, \lambda)}(x) f_{k, m}^{(B, \gamma)}(y) \eta^{k} t^{n-p k} \\
& =\left(1-t^{m}\right)^{-A} e^{-\frac{x t \lambda}{1-t^{m}}}\left(1-\eta^{m}\right)^{-B} e^{-\frac{y \eta \gamma}{1-\eta^{m}}}
\end{aligned}
$$

where $|\eta|<1$

Now, we obtain the following class of bilinear generating function for the $g_{n, m}^{(A, \lambda)}(x)$.

Corollary 4. If

$$
\Lambda_{\mu, \nu}^{n, p}(x ; y ; z):=\sum_{k=0}^{[n / p]} a_{k} g_{n-p k, m}^{(A+B, \lambda)}(2 x) g_{\mu+\nu k, m}^{(C, \gamma)}(y) z^{k},
$$

where $a_{k} \neq 0, n, p \in \mathbb{N}, \mu, v \in \mathbb{N}_{0}, A B=B A$, then we have

$$
\sum_{k=0}^{n} \sum_{l=0}^{[k / p]} a_{l} g_{n-k, m}^{(A, \lambda)}(x) g_{k-p l, m}^{(B, \lambda)}(x) g_{\mu+\nu l, m}^{(C, \gamma)}(y) z^{l}=\Lambda_{\mu, \nu}^{n, p}(x ; y ; z)
$$


provided that each member of (3.10) exists.

Furthermore, for every suitable choice of the coefficients $a_{k}\left(k \in \mathbb{N}_{0}\right)$, if the multivariable function $\Omega_{\mu+\psi k}\left(y_{1}, \ldots, y_{s}\right),(s \in \mathbb{N})$, is expressed as an appropriate product of several simpler functions, the assertions of Theorem 1 and Corollary 1 can be applied in order to derive various families of multilinear and multilateral generating functions for the $f_{n, m}^{(A, \lambda)}(x)$ and $g_{n, m}^{(A, \lambda)}(x)$.

\section{REFERENCES}

[1] R. Aktaş, B. Çekim, and R. Şahin, "The matrix version for the multivariable Humbert polynomials," Math. Notes, Miskolc, vol. 13, no. 2, pp. 197-208, 2012.

[2] B. Çekim, A. Altın, and R. Aktaş, "Some relations satisfied by orthogonal matrix polynomials," Hacet. J. Math. Stat., vol. 40, no. 2, pp. 241-253, 2011.

[3] E. Defez, L. Jódar, and A. Law, "Jacobi matrix differential equation, polynomial solutions, and their properties," Comput. Math. Appl., vol. 48, no. 5-6, pp. 789-803, 2004.

[4] E. Defez and L. Jódar, "Chebyshev matrix polynomials and second order matrix differential equations," Util. Math., vol. 61, pp. 107-123, 2002.

[5] G. B. Djordjević, "On the generalized Laguerre polynomials," Fibonacci Q., vol. 39, no. 5, pp. 403-408, 2001.

[6] N. Dunford and J. Schwartz, Linear Operators. New York: Interscience, 1957, vol. I.

[7] L. Jódar and R. Company, "Hermite matrix polynomials and second order matrix differential equations," Approximation Theory Appl., vol. 12, no. 2, pp. 20-30, 1996.

[8] L. Jódar, R. Company, and E. Navarro, "Laguerre matrix polynomials and systems of second-order differential equations," Appl. Numer. Math., vol. 15, no. 1, pp. 53-63, 1994.

[9] L. Jódar, R. Company, and E. Ponsoda, "Orthogonal matrix polynomials and systems of second order differential equations," Differ. Equ. Dyn. Syst., vol. 3, no. 3, pp. 269-288, 1995.

[10] Z. M. G. Kishka, A. Shehata, and M. Abuldahab, "The generalized Bessel matrix polynomials," J. Math. Comput. Sci., vol. 2, no. 2, pp. 305-316, 2012.

[11] S. Varma, B. Çekim, and F. Taşdelen Yeşildal, "On Konhauser matrix polynomials." Ars Comb., vol. 100, pp. 193-204, 2011.

[12] S. Varma and F. Taşdelen, "Biorthogonal matrix polynomials related to Jacobi matrix polynomials," Comput. Math. Appl., vol. 62, no. 10, pp. 3663-3668, 2011.

\section{Author's address}

\section{Bayram Çekim}

Gazi University, Faculty of Science, Department of Mathematics, Teknik Okullar TR-06500, Ankara, Turkey

E-mail address: bayramcekimegazi.edu.tr 